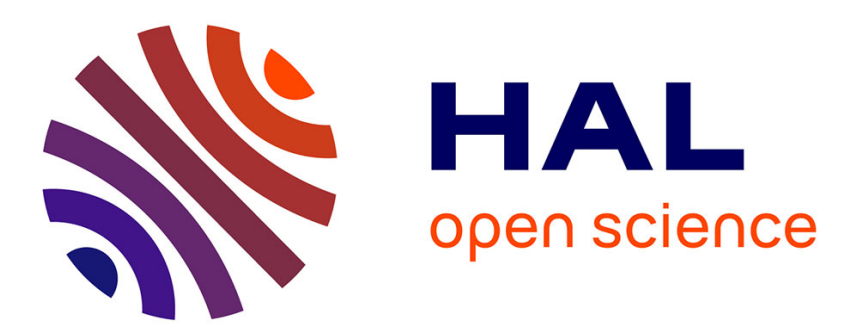

\title{
A remote observer and controller with adaptation to the network Quality of Service
}

\author{
Wenjuan Jiang, Alexandre Kruszewski, Jean-Pierre Richard, Armand \\ Toguyeni
}

\section{- To cite this version:}

Wenjuan Jiang, Alexandre Kruszewski, Jean-Pierre Richard, Armand Toguyeni. A remote observer and controller with adaptation to the network Quality of Service. ECC'09, 10th European Control Conf., EUCA-IFAC-IEEE, Aug 2009, Budapest, Hungary. pp.666. hal-00386276

\section{HAL Id: hal-00386276 https://hal.science/hal-00386276}

Submitted on 20 May 2009

HAL is a multi-disciplinary open access archive for the deposit and dissemination of scientific research documents, whether they are published or not. The documents may come from teaching and research institutions in France or abroad, or from public or private research centers.
L'archive ouverte pluridisciplinaire HAL, est destinée au dépôt et à la diffusion de documents scientifiques de niveau recherche, publiés ou non, émanant des établissements d'enseignement et de recherche français ou étrangers, des laboratoires publics ou privés. 


\title{
A remote observer and controller with adaptation to the network Quality of Service
}

\author{
Wenjuan Jiang, Alexandre Kruszewski, Jean-Pierre Richard and Armand Toguyeni
}

\begin{abstract}
This work is devoted to the remote feedback control of a linear process with "Internet in the loop". In such a networked control situation, variable and unpredictable delays arise, which may decrease the global performance and, in the extreme, destabilize the system. Our aim is to obtain the best performance (here, the exponential stability rate) despite the variation of the network QoS (quality of service). The considered application is based on a Master-Slave structure. The Slave is a light mobile robot, that receives control data and sends its sampled output (position) via a UDP protocol (indeed, packet re-emission of an old output sample is not needed). A Master computer realizes the remote control, the design of which is based on a remote observer achieving a state prediction of the Slave despite the variable delays and possible packet losses. Using time-stamped packets, the Master also detects the network QoS by estimating the variable time delays. It then uses this information so to adapt its observer/controller gains and guarantee the best possible decay rate. The design of this gain scheduling strategy relies on Lyapunov-Krasovskii functionals with an LMI optimization. Experimental results are provided.

Index Terms-Remote control, Switching signal, Exponential stability, Linear time-delay system, LMIs, Internet, UDP, Robot.
\end{abstract}

\section{INTRODUCTION}

Networked Control System (NCS) is a type of closedloop control system with real-time communication networks imported into the control and feedback channels. The signals exchanged among the system's components are in the form of information packets through a network. Network induced delays vary depending on the network hardware, on the different protocols, on the traffic load... In some cases, such as in token ring local area network, the time-delay is bounded; for other networks like Internet, the time-delay is unbounded and varying.

On the basis of Internet and Ethernet fast development, remote control has been widely used in industrial, communicational, medical systems, to cite a few. However, alongside the advantage of low costs, the Internet inevitably brings problems to the closed-loop controlled system, such as delay variation, data-packets loss [1] and disorder, which can cause poor performance, instability or danger (see for instance chapiter 1 of [2],[3] and the references herein).

How to diminish the effect of time delay in the remote system is critical in the system design. The main solution can split into two (combinable) strategies [2], [4]: 1) increase the network performances (QoS) [5] or 2) design an adapted control that can compensate the network influence.

Wenjuan Jiang, Alexandre Kruszewski, Jean-Pierre Richard and Armand Toguyeni are with LAGIS CNRS UMR 8146, Ecole Centrale de Lille, BP 48, 59651 Villeneuve d'Ascq Cedex, France. Jean-Pierre Richard is also with the project ALIEN, INRIA. wenjuan.jiang@ec-lille.fr, Alexandre.Kruszewski@ec-lille.fr,

jean-pierre.richarddec-lille.fr, armand.toguyeni@ec-lille.fr Council).

The work is partially supported by the CSC (China Scholarship
A variety of stability and control techniques have been developed for general time delay systems [6], [7]. But some of these results are based on simplifying assumptions (for instance, the delays are constant) or lead to technical solutions that decrease the performances. In fact, to consider the time delay as constant [8], [9], [10] is actually unrealistic due to the dynamic character of the network. A delay maximizing strategy [11], [12], [13] ("virtual delay", "buffer", or "waiting" strategy) can be carried out so as to make the delay become constant and known. This requires the knowledge of the maximum delay values $h_{m}$. However, it is obvious that maximizing the delay up to its largest value decreases the speed performance of the remote system. Several results are limited to time-delay whose value is less than the sensor and controller sampling periods [14], [15]. In the Internet case, this constraint leads to increase the sampling periods up to the maximal network delay, which may be constraining for high dynamic applications.

Note that, in the Internet case, the network delays cannot be modeled nor predicted. Because of this lack of knowledge, predictor-based control laws [16] cannot be applied. Moreover, the (variable) transmission delays are asymmetric, which means that the delay $h_{1}(t)$ from Master to Slave (shortly, M-to-S), and the return one (S-to-M) $h_{2}(t)$ normally satisfy $h_{1}(t) \neq h_{2}(t)$. In [1] and [17], no such assumptions were needed. The former one used Lyapunov-Razumikhin approach and did not consider the performances but stability.

As in [17], [18], our aim is to ensure suitable stabilization and speed performances, i.e. exponential stabilization, despite the dynamic variations of the network. The first improvement is made by considering the QoS which is estimated by measuring the communication delays and adapting the controller gains (like in [18]). Trough the Lyapunov-Krasovskii approach which is generally less conservative than the $L-R$ one, we derive some stabilization conditions. Unlike [18], the controller design consists in the resolution of a unique LMI problem (Linear Matrix Inequality) which are easily solved using interior-pointlike algorithm [19]. These properties allows making the design independent on the search algorithm.

The remainder of the paper is organized as follows: The features of the feedback control system are described in Section II. In Section III, problem formulation and preliminaries on the stability of switched systems and time-delay systems are presented, followed by the experimental results from an application on a remote controlled robot.

\section{FEATURES OF THE REMOTE SYSTEM}

\section{A. Description of the remote system}

The remote system is based on Master-Slave structure. As in many M-S systems, the Slave is a low energy consumption system with a limited computation power, so the work of the Slave PC is simplified and the control and observation complexity is concentrated on the Master. This structure makes our theory and application easily to be adapted to sensor and actuator network control systems where the sensors usually do not have powerful capability of calculation. The main features of the system refer to Fig.1. In the system, the robot Miabot 


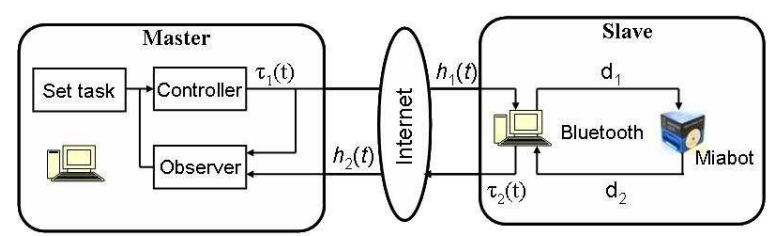

Fig. 1. Structure of the global system

of the company Merlin Systems Corp. Ltd together with a PC serves as the Slave.

The transmission protocol UDP is applied to communicate the data between Master and Slave. In order to know the instant when the positions of the robot have been measured, the Master and the Slave are synchronized before the system starts by the way of NTP (Network Time Protocol) [20] and time stamps are added to the data packets.

\section{B. The four delay sources}

In such a situation, the variable delays come from: 1) the communication through the Internet; 2) the data-sampling; 3) time delay of the Bluetooth and 4) the possible packet losses. In the sequel, $h_{1}(t)$ and $h_{2}(t)$ denote the communication delays and $\tau_{1}(t)$ and $\tau_{2}(t)$ include the sampling delays and possible packet losses. The total Master-to-Slave delay $\delta_{\text {con }}(t)$ results from the addition of $h_{1}(t)$ and $\tau_{1}(t)$. The same phenomenon stands for the Slave-to-Master delay which is denoted $\delta_{o b s}(t)$. In order to simplify the observer design, a buffer is added in the M-S communication. This ensures that the observer "knows" when the control will be applied. Whereas, on the side of the Master, the measurement information is directly applied to the observer.

\section{Effects of time-delay on the performance}

The time-delay of the Internet varies a lot especially between the rush hour and idle time periods. In order to guarantee the exponential stabilization, we have to choose the maximum admissible time-delay, whereas most of the time, the timedelay is much smaller. In other words, the performance is decreased. That is to say, increasing the maximum time-delay means decreasing the performance of the system.

In order to enhance the performance and make the system adaptable to the varying time-delay of the Internet, we propose a switching controller design. In our system, two subsystems are considered, which switch according to the value of time-delay. The definition of the extrema of two modes of time-delay is given as $\left[\sigma_{\min }^{1}, \sigma_{\max }^{1}\right]$ and $\left[\sigma_{\min }^{2}, \sigma_{\max }^{2}\right]$, where $\sigma_{\max }^{1}=\sigma_{\min }^{2}$. Of course, for greater delay values, the performance cannot be guaranteed anymore and an alternative solution has to be considered. In our system, we give a command for the robot to stop until the communication comes back to a sufficient quality.

\section{EXPONENTIAL STABILIZATION OF THE SWITCHED SYSTEM}

\section{A. Problem formulation and preliminaries}

Consider the Slave as a linear system. It is described by the following form:

$$
\left\{\begin{array}{l}
\dot{x}(t)=A x(t)+B u\left(t-\delta_{\text {con }}(t)\right) \\
y(t)=C x(t)
\end{array}\right.
$$
1.

where $i=1,2, \delta_{\text {con }}(t)=\delta+\eta_{\text {con }}(t),\left|\eta_{\text {con }}(t)\right| \leq \mu$ and $\dot{\delta}_{\text {con }}(t) \leq$

This allows for using of a polytopic formulation of the variable delays [17]. In order to guarantee the closed-loop performance whatever the delay variation, the exponential stability with the rate $\alpha$ can be achieved. In other words, there must be a real $\kappa \geq 1$ so that the solution $x\left(t ; t_{0}, \phi\right)$ starting at any time $t_{0}$ from any initial function $\phi$ satisfies: $\left\|x\left(t ; t_{0}, \phi\right)\right\| \leq$ $\kappa\|\phi\|_{c} e^{-\alpha\left(t-t_{0}\right)}$. In this paper, it is achieved using the following state feedback and observer:

$$
\begin{gathered}
u(t)=-K_{i} \hat{x}+k y_{c}, \\
\begin{cases}\dot{\hat{x}}(t)=A \hat{x}(t)+B u\left(t-\delta_{\text {con }}(t)\right) \\
-L_{i}\left(y\left(t-\delta_{\text {obs }}(t)\right)-\hat{y}\left(t-\delta_{\text {obs }}(t)\right)\right), \\
\hat{y}(t)=C \hat{x}(t),\end{cases}
\end{gathered}
$$

$i=1,2$ corresponding the two switching periods. $y_{c}$ being the desired set point and $k$ a gain ensuring an unitary static gain for the closed loop.

Because the observer "knows" exactly when the control will be applied, one has the separation principle, one can divide the analysis of the global stabilization into two smaller problems: the observer design and the controller design.

\section{B. Stability conditions for time-delay system}

Consider the time-delay system

$$
\dot{x}(t)=A x(t)+A_{d} x(t-\delta(t)),
$$

where $\delta(t)$ is a fast varying bounded time-delay.

In order to guarantee some performances $(\alpha$ exponential stability), one applies the transformation $x_{\alpha}(t)=e^{\alpha t} x(t), \alpha>0$ to the system and gets the following equation:

$$
\dot{x}_{\alpha}(t)=(A+\alpha I) x_{\alpha}(t)+e^{\alpha \delta(t)} A_{d} x_{\alpha}(t-\delta(t)),
$$

To prove the exponential stability of the system with a decay rate $\alpha$ in the time-delay zone $\delta(t) \in\left[\sigma_{\min }, \sigma_{\max }\right]$, We use the following $L-K$ functional with descriptor representation [21]:

$$
\begin{aligned}
V\left(x_{\alpha}(t)\right)= & \bar{x}_{\alpha}^{T}(t) E P \bar{x}_{\alpha}(t)+\int_{-\delta}^{0} \int_{t+\theta}^{t} \dot{x}_{\alpha}^{T}(s) R \dot{x}_{\alpha}(s) d s d \theta+ \\
& \int_{t-\delta}^{t} \delta_{\alpha}^{T}(s) S x_{\alpha}(s) d s+ \\
& \int_{-\mu}^{\mu} \int_{t+\theta-\delta}^{t} \dot{x}_{\alpha}^{T}(s) R_{a} \dot{x}_{\alpha}(s) d s d \theta
\end{aligned}
$$

where $E=\operatorname{diag}\left\{I, 0_{(2 \times 2)}\right\}$ and $\mu=\left(\sigma_{\min }+\sigma_{\max }\right) / 2, \delta=\left(\sigma_{\max }-\right.$ $\left.\sigma_{\min }\right) / 2$.

Theorem 1: [17] Suppose that, for a given scalar $\alpha$, there exists some matrices with the appropriate size $0<P_{1}, P_{2}, P_{3}, S$, $Y_{1}, Y_{2}, Z_{1}, Z_{2}, Z_{3}, R, R_{a}$, such that the following LMI conditions are satisfied for $i=1,2$ :

$$
\begin{gathered}
{\left[\begin{array}{ccc}
\Psi & {\left[\begin{array}{c}
\beta_{i} P_{2}^{T} A_{d}-Y_{1} \\
\beta_{i} P_{3}^{T} A_{d}-Y_{2}
\end{array}\right]} & \mu \beta_{i}\left[\begin{array}{c}
P_{2}^{T} A_{d} \\
P_{3}^{T} A_{d}
\end{array}\right] \\
0 & -S \\
* & -S & -\mu R_{a},
\end{array}\right]<0} \\
\left.* \begin{array}{ccc}
R & Y_{1} & Y_{2} \\
* & Z_{1} & Z_{2} \\
* & * & Z_{3}
\end{array}\right] \geq 0 \\
P=\left[\begin{array}{cc}
P_{1} & 0 \\
P_{2} & P_{3}
\end{array}\right]
\end{gathered}
$$

$$
\begin{array}{ll}
{[\Psi]_{11}=} & P_{2}^{T}(A+\alpha I)+(A+\alpha I)^{T} P_{2}+S+\delta Z_{1}+Y_{1}+Y_{1}^{T}, \\
{[\Psi]_{12}=} & P_{1}-P_{2}^{T}+P_{3}^{T}(A+\alpha I)^{T}+\delta Z_{2}+Y_{2}, \\
{[\Psi]_{22}=} & -\left(P_{3}+P_{3}^{T}\right)+\delta Z_{3}+2 \mu R_{a}+\delta R .
\end{array}
$$

where $\beta_{i}$ are defined by:

$$
\beta_{1}=e^{\alpha(\delta-\mu)}, \quad \beta_{2}=e^{\alpha(\delta+\mu)},
$$

Then, the system is exponential stable with a decay rate $\alpha$. In the following, the conditions ensuring the decreasing of the Lyapunov functional (6) considering the delay $\delta(t) \in\left[\sigma_{\min }, \sigma_{\max }\right]$ are noted: $L M I_{\text {stab }}\left(P_{1}, P_{2}, P_{3}, S, Y_{1}, Y_{2}, Z_{1}, Z_{2}, Z_{3}, R, R_{a}, A d, \delta, \mu, \alpha\right)$. 


\section{Observer design}

We define the error vector between the estimated state $\hat{x}(t)$ and the present system state $x(t)$ as $e(t)=x(t)-\hat{x}(t)$. From (1) and (3), this error is ruled by:

$$
\dot{e}(t)=A e(t)+L C e(t-\delta(t)) .
$$

Applying the previous theorem, choosing $P_{3}=\varepsilon P_{2}$ and applying the bijective transformation $W=P_{2}^{T} L$, then we get the following result.

Theorem 2: Suppose that, for a given scalars $\alpha$, and $\varepsilon$, there exists some matrices $0<P_{1}, P_{2}, S, Y_{1}, Y_{2}, Z_{1}, Z_{2}, Z_{3}, R, R_{a}$ and a matrix $W$ with appropriate dimensions, such that the following LMI conditions are satisfied for $i=1,2$ :

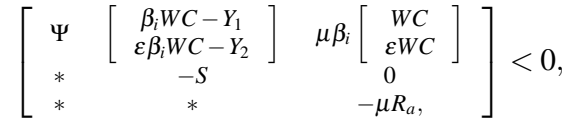

$$
\begin{aligned}
& {[\Psi]_{11}=P_{2}^{T}(A+\alpha I)+(A+\alpha I)^{T} P_{2}+S+\delta Z_{1}+Y_{1}+Y_{1}^{T},} \\
& {[\Psi]_{12}=P_{1}-P_{2}^{T}+\varepsilon P_{2}^{T}(A+\alpha I)^{T}+\delta Z_{2}+Y_{2} \text {, }} \\
& \Psi_{22}=-\varepsilon\left(P_{2}+P_{2}^{T}\right)+\delta Z_{3}+2 \mu R_{a}+\delta R
\end{aligned}
$$

and equations (8) and (11).

Then, the observer gain:

$$
L=\left(P_{2}^{T}\right)^{-1} W
$$

make the estimation error exponentially converging to zero, with the decay rate $\alpha$.

In the following, the conditions ensuring the exponential stability an observer considering the delay $\delta(t) \in\left[\sigma_{\min }, \sigma_{\text {max }}\right]$ are noted:

$L M I_{o b s}\left(P_{1}, P_{2}, \varepsilon, S, Y_{1}, Y_{2}, Z_{1}, Z_{2}, Z_{3}, R, R_{a}, W, \delta, \mu, \alpha\right)$.

\section{Controller design}

We first consider a controller $u=K \hat{x}(t), i=1,2$, i.e. the ideal situation $e(t)=0, x(t)=\hat{x}(t)$ and:

$$
\dot{x}(t)=A x(t)+B K x(t-\delta(t)) .
$$

Applying the previous theorem, choosing $P_{3}=\varepsilon P_{2}$, applying the bijective transformation $M=K P_{2}$ and some matrix manipulations (congruence), then we get following result.

Theorem 3: Suppose that, for some positive scalars $\alpha$ and $\varepsilon$, there exists some matrices $0<P_{1}, P_{2}, S, Y_{1}, Y_{2}, Z_{1}, Z_{2}, Z_{3}$, $R, R_{a}$, and a matrix $M$, with appropriate dimensions such that the following $\mathbf{L M I}$ conditions are satisfied for $i=1,2$ :

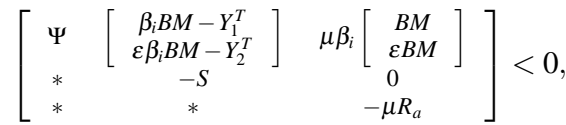

$$
\begin{aligned}
& {[\Psi]_{11}=(A+\alpha I) P_{2}+P_{2}^{T}(A+\alpha I)^{T}+S_{i}+\delta Z_{1}+Y_{1}+Y_{1}^{T},} \\
& {[\Psi]_{12}=P_{1}-P_{2}+\varepsilon P_{2}^{T}(A+\alpha I)^{T}+\delta Z_{2}+Y_{2}} \\
& {[\Psi]_{22}=-\varepsilon\left(P_{2}+P_{2}^{T}\right)+\delta Z_{3}+2 \mu R_{a}+\delta R .}
\end{aligned}
$$

and equations (8) and (11),

Then, the control gain:

$$
K=M P_{2}^{-1}
$$

exponentially stabilizes the system (1) with the decay rate $\alpha$.

In the following, the conditions ensuring the exponential stabilization of the system considering the delay $\delta(t) \in\left[\sigma_{\min }, \sigma_{\max }\right]$ are noted:

$\operatorname{LMI}_{\text {con }}\left(P_{1}, P_{2}, \varepsilon, S, Y_{1}, Y_{2}, Z_{1}, Z_{2}, Z_{3}, R, R_{a}, M, \delta, \mu, \alpha\right)$.

\section{E. The switched control case}

Now let consider the system (1), the switching controller (2) and the switching observer (3). The mode $i$ is active when the time-delay belongs to $\left[\sigma_{\min }^{i}, \sigma_{\max }^{i}\right], i=1,2$, where $\left(\sigma_{\max }^{1}=\sigma_{\min }^{2}\right)$.

Our design strategy is divided into two parts. The first one ensures the uniform stability of the switching closed loop by finding a common Lyapunov-Krasovskii functional for all gains. The second part guarantees some performances $\alpha_{i}$ for the mode $i$ when the delay $\delta_{\text {con }}(t) \in\left[\sigma_{\text {min }}^{i}, \sigma_{\text {max }}^{i}\right]$. The obtained performance indexes are expected to be better than considering only one mode.

The conditions ensuring the first part is given by:

1) $L M I_{c o n}\left(P_{1}^{c}, \ldots, R_{a}^{c}, M_{i}, \delta, \mu, \alpha=0\right), \forall i \in\{1,2\}$

2) $L M I_{o b s}\left(P_{1}^{o}, \ldots, R_{a}^{o}, W_{i}, \delta, \mu, \alpha=0\right), \forall i \in\{1,2\}$

with $\mu$ and $\delta$ corresponding to the global bounds of the system (1) delay. The second part is guaranteed by:

3) $L M I_{c o n}\left(P_{1 i}^{c}, P_{2}^{c}, \varepsilon_{i}^{c}, S_{i}^{c}, \ldots, R_{a i}^{c}, M_{i}, \delta_{i}, \mu_{i}, \alpha_{i}^{c}\right), \forall i \in\{1,2\}$

4) $L M I_{o b s}\left(P_{1 i}^{o}, P_{2}^{o}, \varepsilon_{i}^{o}, S_{i}^{o}, \ldots, R_{a i}^{o}, W_{i}, \delta_{i}, \mu_{i}, \alpha_{i}^{o}\right), \forall i \in\{1,2\}$

with $\mu_{i}=\left(\sigma_{\text {min }}^{i}+\sigma_{\text {max }}^{i}\right) / 2, \delta_{i}=\left(\sigma_{\text {max }}^{i}-\sigma_{\text {min }}^{i}\right) / 2$.

Solving the problems 1) and 3) simultaneously achieves the state feedback design. Thanks to the choice of a common matrix $P_{2}^{c}$ for 1) and 3), the design problem becomes linear. This allows the use of standard LMI solver [19]. The same remark can be done for the observer part. It is only needed to solve 2) and 4). Thanks to the separation principle, the problems $1 \& 3$ and 2 \& 4 can be solved independently.

The choice of these common matrices induces some conservatism but allows keeping the problem solving independent on the algorithm chosen. Only the scalars $\varepsilon_{i}$ have to be chosen.

The next subsection shows the interest of these properties.

\section{F. Comparison with the previous approach}

Considering the theorems in [18], the main flaw is the necessity to find the controller gains (control and observation) and then check the uniform stability.

The observer gains are obtained by solving the following problem without any common $P_{2}$ matrix.

1) $L M I_{o b s}\left(P_{1 i}, \mathbf{P}_{2 i}, \varepsilon_{i}, S_{i}, \ldots, R_{a i}, W_{i}, \delta_{i}, \mu_{i}, \alpha_{i}\right), \forall i \in\{1,2\}$

then the uniform stability is checked by finding a common

L-K function for all modes and for all admissible delays:

2) $L M I_{\text {stab }}\left(P_{1}, P_{2}, \ldots, R, R_{a}, A_{d i}=L_{i} C, \delta, \mu, \alpha=0\right), \forall i \in\{1,2\}$, $K_{i}=\mathbf{P}_{2 i}^{-T} L_{i}$.

The same way is used for the state feedback design.

The flaw of this approach is due to the sequential way to find the gains. Finding a solution for the problem 1) is easy but there is no guarantee to find a common $L-K$ functional ensuring the uniform stability (i.e. problem 2 is not always feasible).

To illustrate this weakness, let consider the following model for the slave:

$$
\left\{\begin{array}{l}
\dot{x}(t)=\left[\begin{array}{cc}
0 & 1 \\
0 & -10
\end{array}\right] x(t)+\left[\begin{array}{c}
0 \\
0.024
\end{array}\right] u\left(t-\delta_{\text {con }}(t)\right) \\
y(t)=\left[\begin{array}{ll}
1 & 0
\end{array}\right] x(t) .
\end{array}\right.
$$

The controller considers two zones of delays $\left[\sigma_{\min }^{i}, \sigma_{\max }^{i}\right]$ where $\sigma_{\text {min }}^{1}=0, \sigma_{\text {max }}^{1}=\sigma_{\text {min }}^{2}=0.09$ and $\sigma_{\text {max }}^{2}=0.5$.

Let consider the following performance indexes for the observation: $\alpha_{1}^{o}=2.2$ and $\alpha_{2}^{o}=1$. The sequential approach is feasible and gives some gains. Now if we consider an easier problem with $\alpha_{1}^{o}=2.2$ and $\alpha_{2}^{o}=0$ (if there is a solution for $\alpha_{2}^{o}=1$ then the same solution ensures $\alpha_{2}^{o}=0$ ), the first step of the sequential approach gives some gains but the uniform stability step fails.

This enlightens the fact that the feasibility of the problems depends on the tuning of the LMI solver.

The second flaw appears when we try to maximize the performances. For example: after trying to maximize the values of the decay rates step by step from $\alpha=0$ to $\alpha=\alpha_{\max }$, in the 
two zones, one obtains for the control $\alpha_{1}^{c}=0.1$ and $\alpha_{2}^{c}=0.2$, whereas there is a solution for: $\alpha_{1}^{c}=3.6$ and $\alpha_{2}^{c}=1$. This shows that the result depends on the search direction.

Considering the "one shot algorithm" presented in the previous subsection, we have removed this dependency on the values of $\alpha$ and the solution does not depend anymore on the direction of search. On the same example, it gives directly $\alpha_{1}^{c}=3.1$ and $\alpha_{2}^{c}=1$.

The main weakness of the new approach is the conservatism brought (the $\alpha_{1}^{c}=3.1$ is less than the one found by [18] ) by the choice of a common matrix $P_{2}$ in the Lyapunov-Krasowskii functionals.

\section{EXPERIMENTS}

The experiments are done on two computers separated from about 40 kilometers away (Fig. 1). The Master program runs on the remote computer with an advanced computing capability, the Slave one on the local one which also communicates with the Miabot by Bluetooth.

\section{A. The structure of the Master}

In order to implement the model for the remote control system, a four-thread program is designed to fulfill the functions of Controller and Observer in Fig.1, while the explication of all the parameters refers to [18].

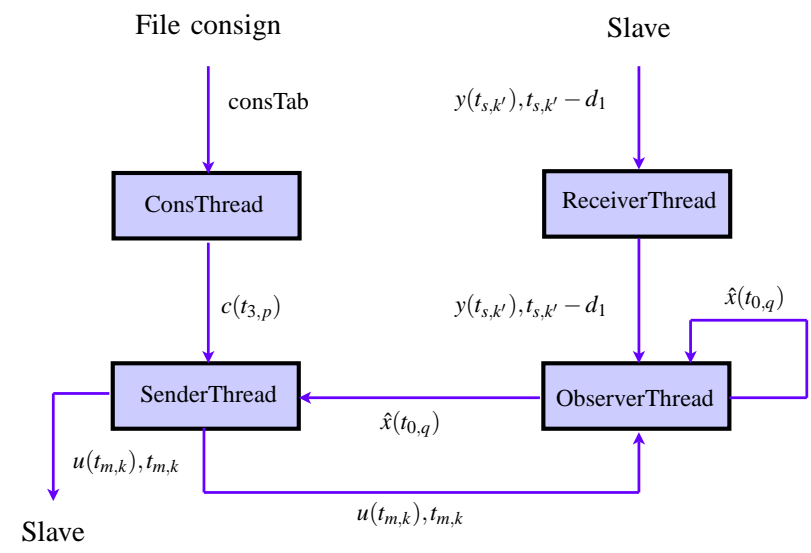

Fig. 2. Structure of the Master

These four threads are concurrently working as showed in Fig.2. (a) ConsThread is a periodic thread which gets the set points of the trajectory. (b) SenderThread is also a periodic thread which calculates the control data for sending out to the Slave. (c) ReceiverThread is a event-driven thread to receive controls from the Slave. (d) ObserverThread is the most important part of the program. It mainly serves as the Observer in the system model. Its main task is to estimate periodically the present position and speed of the remote motor.

\section{B. The structure of the Slave}

The Slave does not need power computation abilities, because it just needs to communicate with the Master and the Miabot. As we can see from Fig.3, this program is divided into two threads: ReceiveThread and SendThread. (a) ReceiveThread is an event-driven thread which is activated by the control data arrived from the Master. (b) SendThread is used to apply the control to the Miabot as well as to get its real position, and then send the data back to the Master. As we need to apply the control data with the time delay of $h_{1 m}$ after the time stamp, a first-sent first-out list list_Y is used to contain the control data temporarily, in which all the nodes are sorted in the order according to the its time stamp. That means the most recent control is inserted at the end of the list.

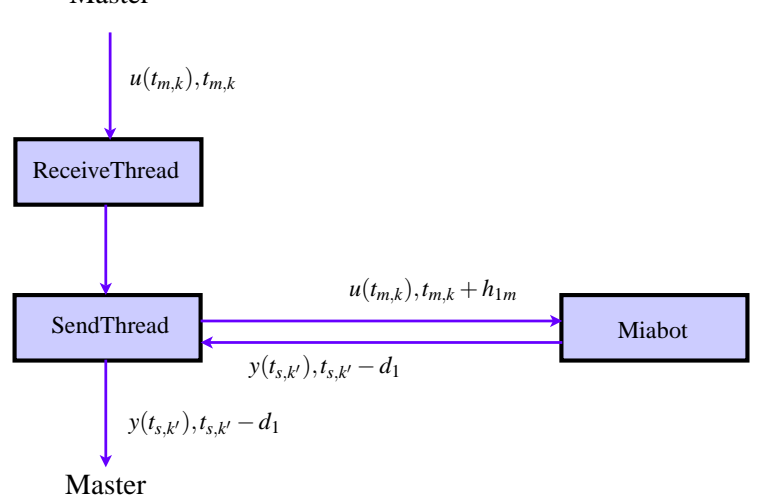

Fig. 3. Structure of the Slave

\section{Experimental study}

According to the identification of the Miabot, when the speed is no more than $2 \mathrm{~m} / \mathrm{sec}$, it can be treated as a linear system and the model is showed in equation (20). The state value is composed of its position and speed, the control is the speed value sent by Master. The RTT (Round-trip-time) between the two PCs have been continuously tested by the protocol ICMP (Internet Control Message) during twenty-four continuous hours and the average value is $52.37 \mathrm{~ms}$ with $4.1 \mathrm{~ms}$ and $577.4 \mathrm{~ms}$ for the minimum and maximum RTT respectively. From these tests, considering also the Bluetooth transmission delays and the sampling delays, we take the value $0.5 \mathrm{sec}$ as the maximum time-delay. If the time-delay is bigger than this value, the packet is treated as lost.

For sake of the simplification of the switching strategy, the same maximum time-delay values are chosen for the controller and the observer. Considering two zones of delay with $\delta_{1}=$ $0.045 \mathrm{sec} \& \mu_{1}=0.045 \mathrm{sec}$ and $\delta_{2}=0.3 \mathrm{sec} \& \mu_{2}=0.2 \mathrm{sec}$. It means that the gains switch when the delay crosses the value of $0.09 \mathrm{sec}$. According to the approach in this paper, the maximum exponential convergence ensuring the global stability are: $\alpha_{1}^{c}=$ $\alpha_{1}^{o}=3.1$ and $\alpha_{2}^{c}=\alpha_{2}^{o}=1$.

The gains $K_{i}$ and $L_{i}(i=1,2)$ are:

$$
\left[\begin{array}{ll}
L_{1} & L_{2}
\end{array}\right]=\left[\begin{array}{ll}
-3.02 & -1.37 \\
-0.27 & -0.36
\end{array}\right],\left[\begin{array}{l}
K_{1} \\
K_{2}
\end{array}\right]=\left[\begin{array}{cl}
-1311 & -129 \\
-682 & -68
\end{array}\right],
$$

\section{Results of remote experiment}

The result is shown in Fig.4, in which the dash-dotted line represents the set values, this is the position that we want to achieve; the dashed-line and straight-line represent respectively the robot's estimated position the real position of the Miabot. Fig.5 illustrate the corresponding switched control signals from Master to Slave. The straight-line curve is the real control while the dashed line and the dots are the controls calculated respectively for the two zones. We can see the switch points according to the values of time-delay. Fig.6 depicts the variable time-delays, which comprise the time-delay of sampling and communication of Bluetooth (we consider it as constant timedelay, here we take the value of $40 \mathrm{~ms}$ ).

On Fig.4, one can notice three kinds of step responses. The first one corresponds to the case where the control switches a lot during the response. In that case, only the global stability is guaranteed. During the second step, only the second zone is active, i.e. only the gains $K_{2}$ and $L_{2}$ are active $(\alpha=1)$. In this case, performances are guaranteed. In the last kind of response, only the first zone is active because the delays are small. In that case, the performances are still better $(\alpha=3.1)$ : the response time is smaller and the damping is greater. 


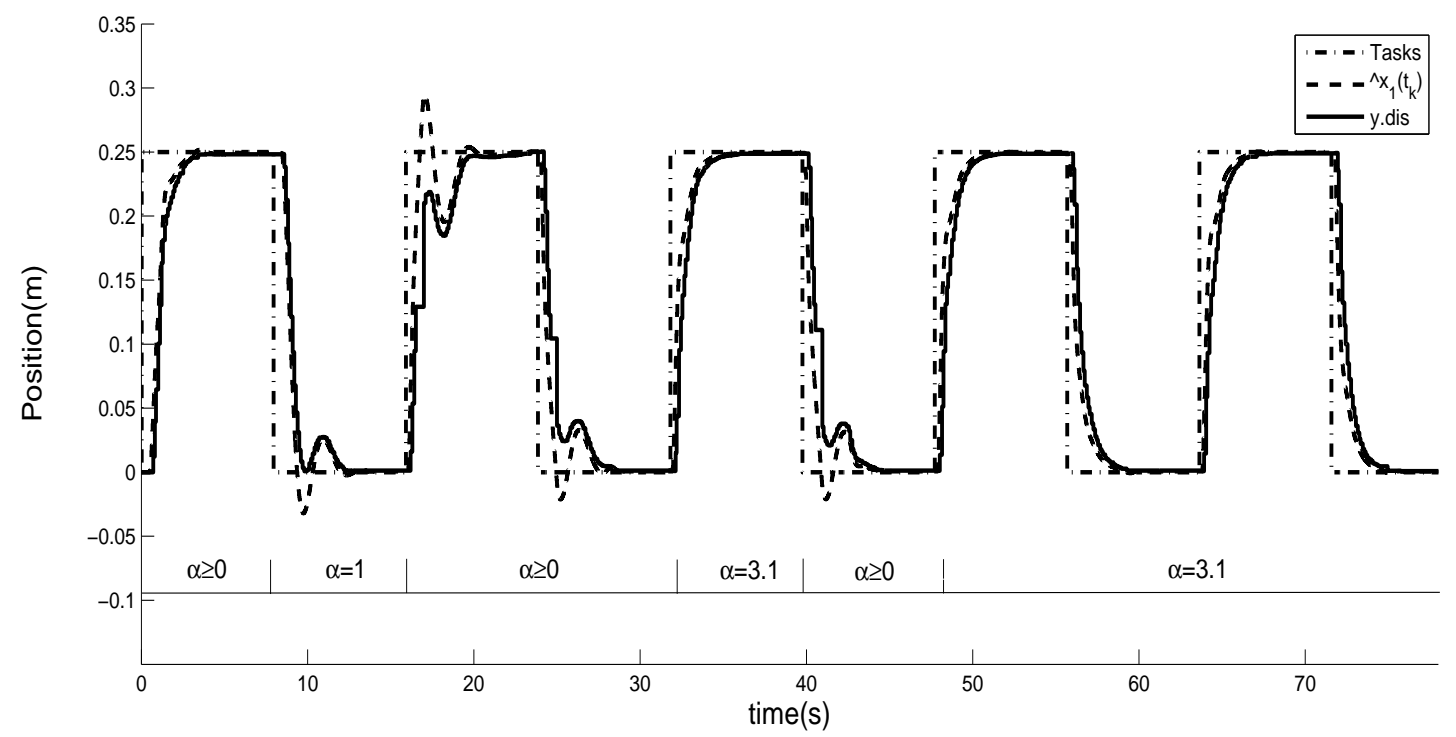

Fig. 4. Results of remote experiment

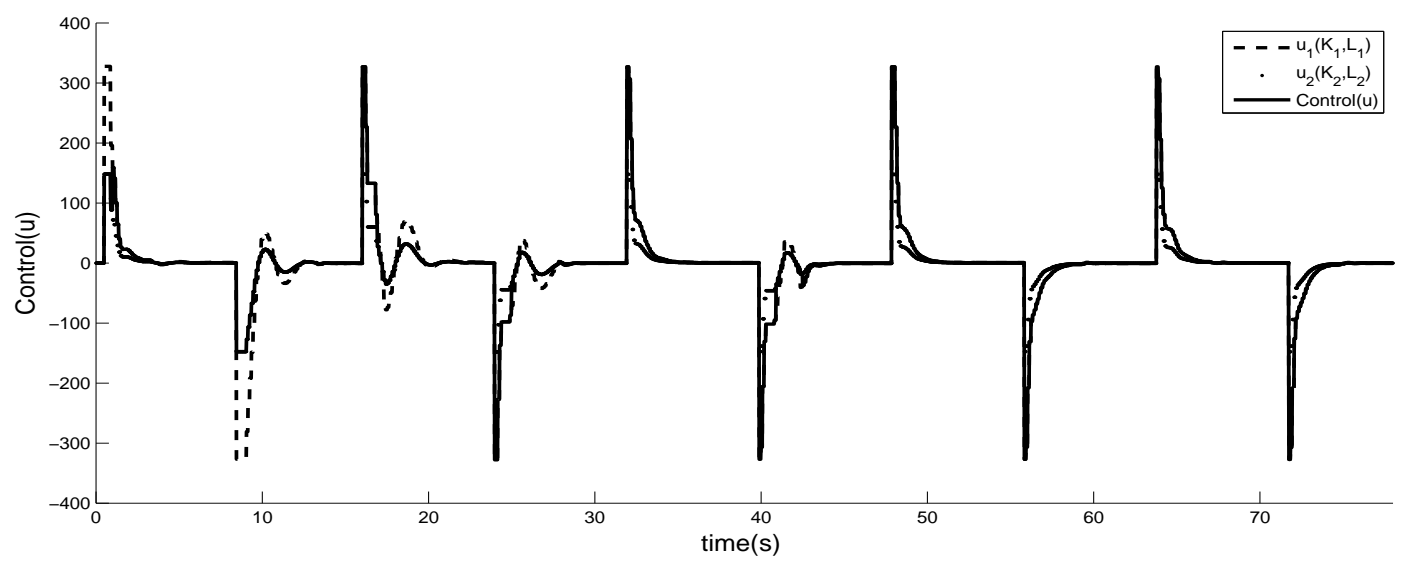

Fig. 5. The corresponding switched control

As it is clearly shown in Fig.4, the global stability of the closed loop is maintained despite the assumption that the timedelay of Bluetooth is treated as a constant one.

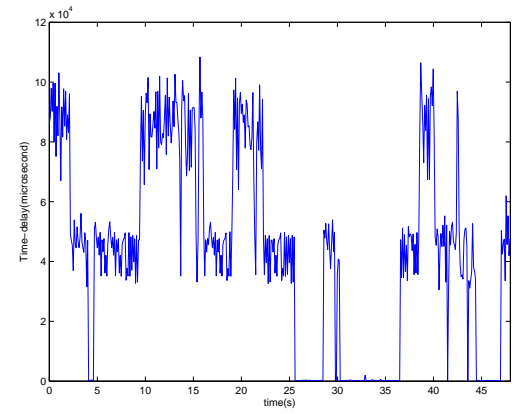

Fig. 6. The corresponding variable time-delays

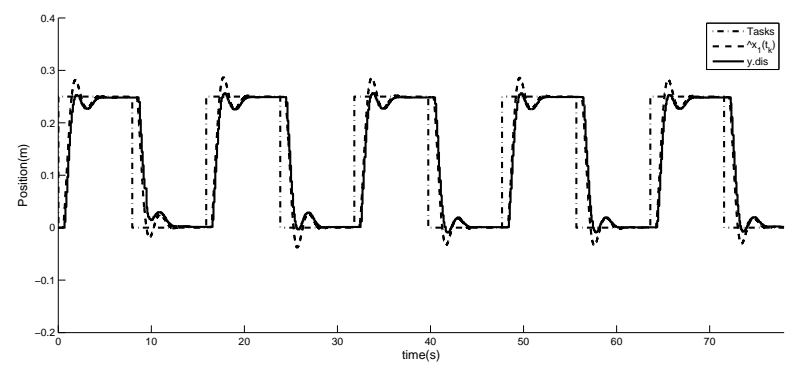

Fig. 7. The results of system without switching

\section{CONCLUSION}

In addition to some theoretical results, an experimental platform has been developed to illustrate the results of the network-based control theory. This platform is able to control 
a Slave robot through a network and joins skills in automatic control, computer science and networks.

The experimental results confirm the theory: 1) The exponential stability is obtained in both the time-delay zones and the uniform stability is guaranteed whatever the zone switches. 2) The experimental performances are showed to be better when considering two modes of time delay instead of one (The experimental results under the same network communication quality but no switching refer to Fig. 7, the value of $\alpha=0.96$ ).

A "one shot algorithm" is proposed to get rid of the search direction when optimizing the value of $\alpha$. In this sense, this result improves previous one [18]. Another trend is to investigate a solution without the input buffer. Without buffer, the input delay will be smaller, ensuring more performances, and the slave will need less memory to run [22].

\section{REFERENCES}

[1] Yu M., Wang L., and Chu T., "An LMI approach to network control systems with data packet dropout and transmission delays," MTNS '04 Proc. of Mathematical Theory Networks and Systems, Leuven, Belgium, 2004.

[2] Richard J.-P. and Divoux T., Systèmes commandés en réseau. Hermes-Lavoisier, IC2, Systèmes Automatisés, 2007.

[3] Georges J.-P., Divoux T., and Rondeau E., "Confronting the performances of a switched ethernet network with industrial constraints by using the network calculus," International Journal of Communication Systems(IJCS), vol. 18, no. 9, pp. 877-903, 2005.

[4] Chiasson J. and Loiseau J.J., Applications of time delay systems. Springer, 2007, vol. 352.

[5] Toguyeni A. and Korbaa O., "Quality of service of internet service provider networks: state of the art and new trends," ICTON Mediterranean Winter Conference, ICTON-MW'07, 2007.

[6] Niculescu S.-I., Delay effects on stability: a robust control approach. Springer, 2001, vol. 269.

[7] Richard J.-P., "Time delay systems: an overview of some recent advances and open problems," Automatica, vol. 39, pp. 1667-1694, 2003.

[8] Huang J.Q. and Lewis F.L., "Neural-network predictive control for nonlinear dynamic systems with time delays," IEEE Transactions on Neural Networks, vol. 14, no. 2, pp. 377-389, 2003.

[9] Fattouh A. and Sename 0 ., " $\mathrm{H}^{\infty}$-based impedance control of teleoperation systems with time delay," 4th Workshop on Time Delay Systems, 92003.

[10] Niemeyer G. and Slotine J.-J., "Towards force-reflecting teleoperation over the internet," IEEE Int. Con. on Robotics \& Automation, 1998.

[11] Lelevé A., Fraisse P., and Dauchez P., "Telerobotics over IP networks: Towards a low-level real-time architecture," IROS'01 International conference on intelligent robots and systems,Maui,Hawaii, October 2001.

[12] Estrada-Garcia H.J., Marquez-Martinez L.A., and Moog C.H., "Master-slave synchronization for two inverted pendulums with communication time-delay," 7th IFAC workshop on time delay systems, Nantes, France, 2007.

[13] Ploplys N.J., Kawka P.A., and Alleyne A.G., "Closed-loop control over wireless networks," IEEE Control Systems Magazine, June 2004.

[14] Chen Z., Liu L., and Yin X., "Networked control system with network time-delay compensation," Industry Applications Conference, Fourtieth IAS Annual Meeting, vol. 4, pp. 2435 - 2440, 102005.

[15] Nilsson J., Bernhardsson B., and Wittenmark B., "Stochastic analysis and control of real-time control systems with random time delay." Automatica, vol. 34, no. 1, pp. 57-64, 1998.

[16] Witrant E., Canudas-De-Wit C., and Georges D., "Remote stabilization via communication networks with a distributed control law," IEEE Transactions on Automatic control, 2007.

[17] Seuret A., Michaut F., Richard J.-P., and Divoux T., "Networked control using gps synchronization," Proc. of ACC06, American Control Conf., Mineapolis, USA, June 2006.

[18] Jiang W.-J., Kruszewski A., Richard J.-P., and Toguyeni A., “A gain scheduling strategy for the control and estimation of a remote robot via internet," The 27th Chinese Control Conference, July 2008.
19] Boyd S., El Ghaoui L., Feron E., and Balakrishnan V., "Linear matrix inequalities in sytem and control theory," SIAM. Studies in Applied Mathematics, 1994.

[20] Mills D.L., "Improved algorithms for synchronizing computer network clocks," IEEE/ACM Transactions On Networking, vol. 3, no. 3, pp. 245-254, June 1995.

[21] Fridman E., "A new lyapunov technique for robust control of systems with uncertain non-small delays," IMA Journal of Mathematical Control and Information, vol. 23, no. 2, pp. 165179, 2006.

[22] Seuret A. and Richard J.-P., "Control of a remote system over network including delays and packet dropout," IFAC World Congress, Seoul, Korea, July 2008. 